\title{
Thermosensitive demineralization hydrogel for water softening: preliminary batch and column experiments
}

\author{
S. Olivero ${ }^{1}$, S. G. J. Heijman ${ }^{1}$, J. P. A. Custers ${ }^{2}$, \\ G. Dascola ${ }^{3}$ \& L. C. Rietveld ${ }^{1}$ \\ ${ }^{I}$ Delft University of Technology, CiTG, The Netherlands \\ ${ }^{2}$ Afira BV, The Netherlands \\ ${ }^{3}$ Politecnico di Torino, Italy
}

\begin{abstract}
Softening remains one of the main problems in industrial and domestic processes. Polyvalent ions such as $\mathrm{Ca}$ and $\mathrm{Mg}$ form poorly soluble salts which precipitate in pipes and heating apparatuses, resulting in potential clogging or efficiency losses in heat exchangers. In a washing process, calcium and other ions can precipitate with the detergent molecules, inhibiting the cleaning action of soaps. Ion-exchange is used as a softening process, but the reinforcement of regulations regarding the discharge of regenerants makes this solution more and more expensive. The present work is meant to introduce experimental and modeling results of a thermosensitive hydrogel based on a LCST polymer, able to soften water at low temperature, and regenerable with low-grade waste heat without the addition of any chemical product. The polyampholytic polymer matter of this research is a semi-IPN (Interpenetrating Polymer Network) which presents a crosslinked polymer matrix comprising PNIPAAm and basic cationic groups, interwoven by a polymeric chain including acidic anionic monomers. In preliminary batch experiments, with full grain size of the exchanger and varying concentration of calcium in the starting synthetic solution, the adsorption parameters according to Freundlich's approach can be measured. Modeling based on LDF-theory using the obtained parameters shows an accurate prediction of the breakthrough curve of subsequent column experiments. The research illustrates lower capacity of the hydrogel than commonly used ion
\end{abstract}


exchangers $\left(0.07 \mathrm{~mol} \mathrm{Ca}^{2+} / \mathrm{Kg}\right.$ of resin), but the results show a good regeneration ability and potential future application of the system.

Keywords: LCST polymers, water softening, thermoregenerable resins, $L D F$.

\section{Introduction}

\subsection{Hard water and softening}

Industrial and drinking purposes have created the need of purification of water coming from natural sources, or even extra-purification of treated water. Hard water multivalent ions such as $\mathrm{Ca}, \mathrm{Mg}$, and $\mathrm{Ba}$ form sparingly soluble salts, inducing scale problems and severe damages and failures in pipes and heating apparatuses, at industrial and domestic scale - i.e. in dishwashers, washing machines, steam irons (Gabrielli et al. [1]). In a washing process, these ions precipitate with the detergent molecules, inhibiting the cleaning action of soaps, contributing to an exaggerated use of detergents (Park et al. [2]).

The removal of the ions responsible for the cited problem is one of the main issues in domestic and industrial applications, and various technologies have been widely and effectively applied: chemical precipitation, ion exchange, nanofiltration, reverse osmosis, and different electromembrane systems (Seo et al. [3]). Nevertheless, the above-mentioned technologies present drawbacks of different entities. The choice of additional chemicals is economically problematic, and restricted for the purpose of drinking water, while results are not always satisfactory. For in situ water softening with ion exchangers, acid solutions and brines are used for resin regeneration resulting in harmful effects on the environment, while legislation all over the world made the disposal of such regenerants more and more of a problem. Membrane technology requires high power consumption and difficult maintenance of the equipments (Seo et al. [3]; Ghizellaoui et al. [4]; Čuda et al. [5]), without leaving out the production of a concentrate difficult to discharge.

Problems due to hard water occur, as already stated, both in large-scale industrial applications and in small-scale applications at the level of households. In most of the processes mentioned, hot water flows are available, and low-grade heat flows are even discharged as waste. The object of the present investigation is the characterization of a demineralization resin developed as a water softener, able to subtract multivalent ions from cold water (below $30^{\circ} \mathrm{C}$ ) and regenerable with warmer water (below $60^{\circ} \mathrm{C}$ ). In this study, batch experiments and a first model based on simple LDF (Linear Driving Force) adsorption model are presented. The column experiments are introduced by describing the set-up and the first series of results.

\subsection{LCST polymers}

In the last few years, advances in biological and medical research requested new polymer materials in order to face different tasks: smart drug delivery (Hatefi and Amsden [6]), DNA separation and sequencing (Mikšík et al. [7]), 
filtration/separation (Helfferich [8]), control of microfluidic flow (Beebe et al. [9]), etc. The polymers in charge of such functions, also called "intelligent polymers", respond to different external stimuli such as $\mathrm{pH}$, salt concentration, electric fields, or temperature. Examples can be found in De et al. [10] for $\mathrm{pH}-$ responsive hydrogels (copolymers of HEMA and acrylic acid with $1 \%$ crosslink) with particles diameter variation of $100 \%$ associated to a $\mathrm{pH}$ change between 4 and 6.

LCST polymers, such as Poly(N-isopropylacrylamide) (poly-NIPAAm), define a class of polymers which undergo a transition from the hydrophilic to hydrophobic state with increasing temperature, exhibiting an inverse temperature dependence of solubility (Deshmukh et al. [11] Taylor and Cerankowski [12]). Heskins and Guillet [13] investigated by the end of the 1960s the effects of specific stimuli on these particular polymers, establishing the lower critical solution temperature (or LCST) for poly-NIPAAm to be $32{ }^{\circ} \mathrm{C}$. At this temperature, on standard conditions such as $\mathrm{pH} 7$ and in the absence of ionic compounds, poly-NIPAAm undergoes a phase transition from soluble to insoluble. Temperature sensitive swelling behaviour is obtained by incorporation of hydrophilic or hydrophobic comonomers into the polymer network (Geever et al. [14]). At temperatures lower than LCST, the polymer chains' hydrophilic surfaces interact with water and the strong hydrogen bonding between the hydrophilic groups and water outweighs the unfavourable exposure of hydrophobic groups to water. The increase of hydrogen bonds between the water molecules surrounding the hydrophobic groups compensate for the free energy increase due to the exposure of the hydrophobic groups. This water molecules structuring stabilizes the hydrophobic groups and makes so that, at temperatures lower than the LCST, the polymer results with good solubility. At higher temperatures, the weakening of the hydrogen bonds drives to a lower water structuring, and therefore an increase interaction between the hydrophobic groups. Eventually, this phenomenon becomes predominant, and the polymer chains shrivel into an insoluble glob as the hydrophobic surfaces interact and the chains become dehydrated leading to a subsequent phase separation (Kanazawa et al. [15]). The collapse of the structure of the polymer is an entropy-driven process, where reduced solubility and mobility of the matrix is compensated by the regained mobility of the water molecules.

\section{Materials and methods}

\subsection{Newly designed process for separating a charged species from an aqueous system: introduction to the softening hydrogel (SH)}

The material matter of this research is included in WO 2009/028944 (Custers et $a l$. [16]), and it refers to a newly designed process for separating charged species from an aqueous solution. The presented polyampholytic polymeric material, from now on presented as softening hydrogel (SH), is thermoregenerable, and suitable for a demineralization process mostly focusing on water softening. Among the various possible configuration of the polymeric system introduced by 
the mentioned patent, the polymer used is a semi-IPN (Interpenetrating Polymer Network) which presents a crosslinked polymer matrix comprising PNIPAAm and basic cationic groups, interwoven by a polymeric chain including acidic anionic monomers.

The process comprises two steps: a first (i) demineralization step and a (ii) regeneration step. During the demineralization, the solution to be softened is put in contact with the hydrogel at a first temperature and the cationic and anionic domains within the hydrogel attract and bond the charged species in the aqueous system. In the second step, the polyampholytic polymer is contacted with a second aqueous system at a second temperature, and the charged species previously loaded on the hydrogel are released and the polymer is ready to restart the loading cycle. The main characteristic differencing the two aqueous systems is the temperature, with the second one higher than the first one, but still remaining lower than $60^{\circ} \mathrm{C}$. The temperature upper-limit is fundamental to distinguish the presented system from what is the working principle of other hybrid copolymers, (i.e. Sharples and Bolto [17]), where regeneration at higher temperatures (around $85^{\circ} \mathrm{C}$ ) and high temperature difference between the loading and the regeneration step (at least $35^{\circ} \mathrm{C}$ ) make them less energetically effective. In contrast to these hybrid copolymers, it is hypothesized that the temperature increase over the LCST induces shrinking of the SH structure, which, in turn, induces an increase in charge density and eventual complexation of the oppositely charged groups of the ampholytic domain, making the neutralization effect easier to obtain, thus lowering the temperature needed for regeneration.

In a first period of the research leading to the development of the resin, the drawn outcomes led to the conclusion that at a temperature lower than the LCST, the positively and negatively charged groups within the hydrogel domain would essentially be neutralized by external anions and cations respectively. When

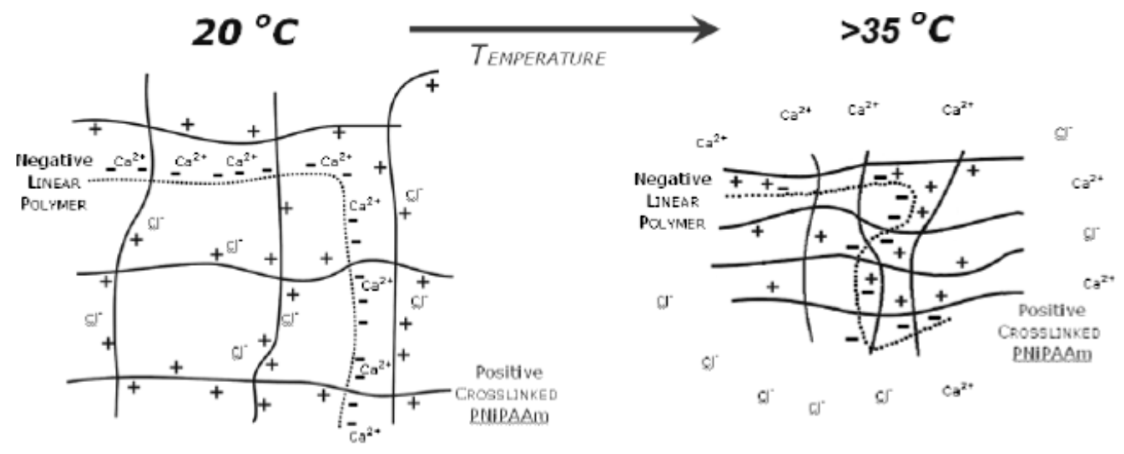

Figure 1: Illustration of the behaviour of the $\mathrm{SH}$ in different aqueous systems: at a temperature lower than the LCST, ions in solution can enter the matrix and reach the binding sites (left part of the figure), while at higher temperature the structure of the hydrogel collapses and ions are expelled in order to maintain the electroneutrality within the polymer itself (right part). 
raising the temperature above the LCST, the "newly-hydrophobic" parts of the polymer matrix would collapse and the charged domains be brought in adjacency, leading to charge neutralization within the ampholytic system itself, and thus to the expulsion of the previously loaded external ions (Figure 1).

Ionic Strength (IS) is considered to play a major role in the sorption process. Influence of IS on the swelling behaviour of hydrogels has been studied by numerous researchers in the last decade (i.e., Cai and Gupta [18], Lee and Chiu [19]), while, on the other hand, both the calcium sorption during the loading process and the hypothesized complexation of the charged groups in the gel matrix during regeneration are supposed to be strongly affected by the change of ionic strength in the solution in contact with the gel itself (i.e. for high ion concentrations the Debye-length or the double layer thickness decreases in systems with charged particles, as stated in Dunn et al. [20]).

\subsection{Equilibrium isotherm and Ca-sorption kinetics}

In order to familiarize with the material's potentials and limitations, a series of batch experiments has been conducted at a constant temperature of $19 \pm 1^{\circ} \mathrm{C}$. The chosen laboratory experiments were expected to give indication on the actual sorption capacity of the hydrogel and information on the kinetics of the sorption process. The main polyvalent ion monitored during the hereby presented research was chosen to be calcium, but the same affinity to magnesium is expected, due to the similar behaviour of the two ions in water.

The distribution of calcium between the hydrogel and the aqueous solution, when the system is in a state of equilibrium, is important to establish the capacity of the material for the hardness ions (McKay [21]). Adsorption isotherms were obtained by mixing a fixed weight of finely grinded (grain size $<100 \mu \mathrm{m}$ ) dry resin $(2 \mathrm{~g})$ with $200 \mathrm{ml}$ of calcium solution of known initial concentrations. Various concentrations of the ion were used and the system was allowed 4 days to reach equilibrium. The $\mathrm{pH}$ of the solution was monitored during the completion of the equilibration process together with the temperature. The system was maintained at the desired temperature by using a thermostatic water bath. All the reagents were analytical grade chemicals. The solutions were prepared by dissolving appropriate amount of $\mathrm{CaCl}_{2} \cdot 2 \mathrm{H}_{2} \mathrm{O}$ (Aldrich Chemical Company, USA) in demineralised water. In order to maintain the IS of the starting solution constant, avoiding the complication due to probable IS interference with the process already mentioned earlier in this paper, an according amount of $\mathrm{NaCl}$ was added to the various solutions. In preliminary experiments, the electric conductivity of a $\mathrm{NaCl}$ solution put in touch with the softening hydrogel did not show significant decrease. This result brought to the conclusion that the affinity of the charged groups to the monovalent ions is low enough for the binding of such ions to be considered negligible (at IS of the starting solution $\leq 0.01 \mathrm{~mol} / \mathrm{l})$. The equilibrium capacity of the hydrogel was calculated using eqn. (1) (Rengaraj et al. [22]), where $q_{e}(\mathrm{~g} / \mathrm{kg}$ of resin) is the equilibrium sorption capacity, $C_{0}$ and $C_{e}(\mathrm{mg} / \mathrm{l})$ the initial and equilibrium concentration of $\mathrm{Ca}^{2+}$ in solution, $V(\mathrm{l})$ the volume and $M(\mathrm{~g})$ the weight of the hydrogel. 


$$
q_{e}=\frac{\left(C_{0}-C_{e}\right) V}{M}
$$

The kinetics of the Ca-sorption process was also studied in a batch experiment. The full-size grains, which were used to fill the column during the column experiments (grain size 250-500 $\mu \mathrm{m}$ ), were put in touch with a rather hard water ( $2 \mathrm{mM} \mathrm{Ca}^{2+}$ solution) at temperature $19 \pm 1{ }^{\circ} \mathrm{C}$. The concentration of calcium was monitored in the solution, and the decrease was considered to be caused only by Ca-sorption in the hydrogel as stated in eqn. (2) (Rengaraj et al. [22]), where $q_{t}$ and $C_{t}$ represent the sorption capacity and the $\mathrm{Ca}^{2+}$ concentration in solution at instant $t$.

$$
q_{t}=\frac{\left(C_{0}-C_{t}\right) V}{M}
$$

Once equilibrium had been reached, the resin was placed into a regenerant solution of demiwater kept at $50^{\circ} \mathrm{C}$. The concentration of $\mathrm{Ca}^{2+}$ ion released in solution was again monitored in time till equilibrium was reached. $\mathrm{Ca}^{2+}$ concentrations were analyzed by atomic absorption spectroscopy (Varian, AA 640 model).

\subsection{Column experiment set-up}

In addition to the batch experiments, a dynamic column set-up was prepared in order to develop meaningful process data. Column studies were considered essential to compare different process configurations and to evaluate the pressure drop due to the presence of the hydrogel bed. The set-up and process parameters can be seen in and Figure 2 and Table 1. The ratio of column diameter to particle diameter was chosen high enough $(>30)$ to minimize wall effects.

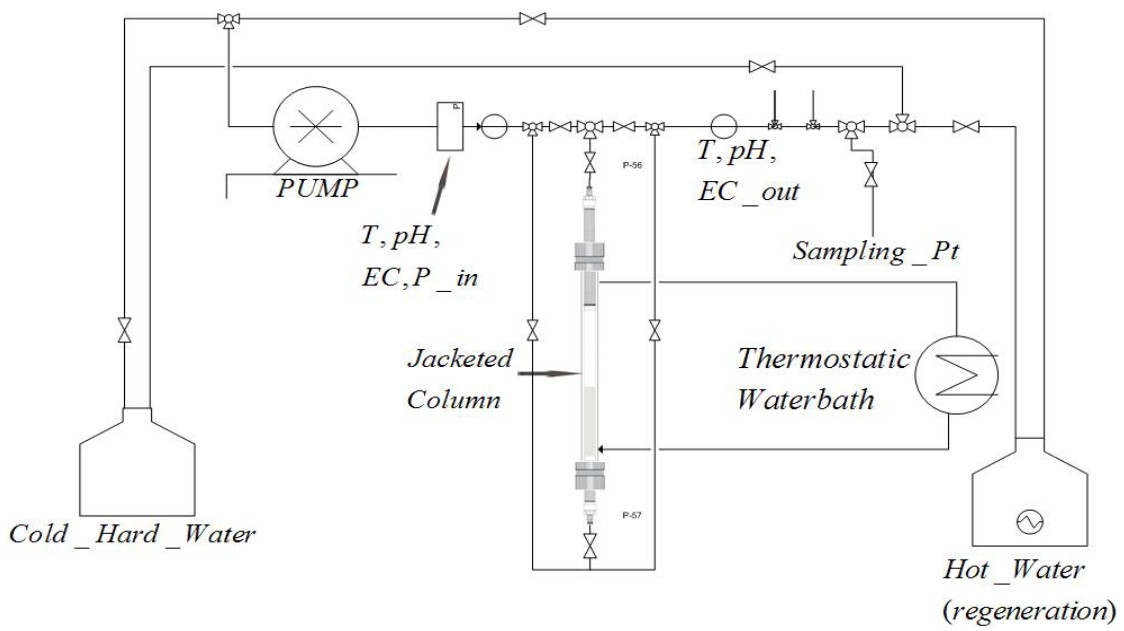

Figure 2: Column experiment set-up. 
Table 1: $\quad$ Process parameters.

\begin{tabular}{|l|c|l|c|}
\hline Inner diameter & $2,62 \mathrm{~cm}$ & Flow Velocity & $1,45 \mathrm{~cm} / \mathrm{min}$ \\
\hline Resin Height & $19,2 \mathrm{~cm}$ & Particle size & $0,25-0,5 \mathrm{~cm}$ \\
\hline Mass of resin & $19,35 \mathrm{~g}$ & EBCT & $13,27 \mathrm{~min}$ \\
\hline Flow-rate & $7,8 \mathrm{ml} / \mathrm{min}$ & Direction of the flow & Downward \\
\hline \multicolumn{2}{|c|}{ Inlet-water composition } & $2 \mathrm{mM} \mathrm{CaCl}$ \\
2 & $+2 \mathrm{mM} \mathrm{NaHCO}_{3}$ & $\mathrm{pH}$ & 7.8 \\
\hline
\end{tabular}

\subsection{Softening kinetics - LDF model}

A great number of complex mathematical models can be found to describe the kinetics of the sorption process. Simplified models, on the other hand, have been introduced mainly to model granular activated carbon filtration in full-scale plants, but examples of their use can be found also regarding ion exchange filters. According to this, the kinetics of the removal of polyvalent-ions from the aqueous system by SH was studied and the results of the batch experiment were interpreted through the model presented by Glueckauf and Coates [23], denominated linear driving force (LDF) model. The accumulation of the dissolved species on the softening hydrogel, can be approximated by eqn. (3) (Peel and Benedek [24])

$$
\frac{\partial q}{\partial t}=k\left[q_{s}(t)-q(t)\right]
$$

in which $t$ is the time in seconds, $q$ is the mean solid phase concentration at time $t, q_{s}$ the solid phase concentration at the particle's external surface (considered in equilibrium with the external concentration), and $k$ the mass transfer coefficient. The LDF model can be used as a relation between the speed of sorption, the sorption isotherm and the LDF-constant $k$ (Heijman et al. [25]), which in the model represents an overall kinetic rate constant covering film diffusion as well as pore diffusion. The speed at which the mass transfer occurs is seen proportional to the difference between the actual concentration and the concentration at equilibrium.

Through simple mathematical substitution, eqn. (3) can be transformed to the exponential eqn. (5) using the Freundlich isotherm in eqn. (4) (rearranged from Wachinski [26]) and the mass balance introduced in eqn. (2). Parameters appearing in eqn. (4) will be further discussed in the next section.

$$
\begin{gathered}
q_{e}=K C_{e}^{n} \\
C_{t}=\left(C_{0}-C_{e}\right) e^{-k t}+C_{e}
\end{gathered}
$$

\section{Results and discussion}

\subsection{Equilibrium isotherm}

In Figure 3 the results of the batch experiments for building the sorption isotherm are presented. 


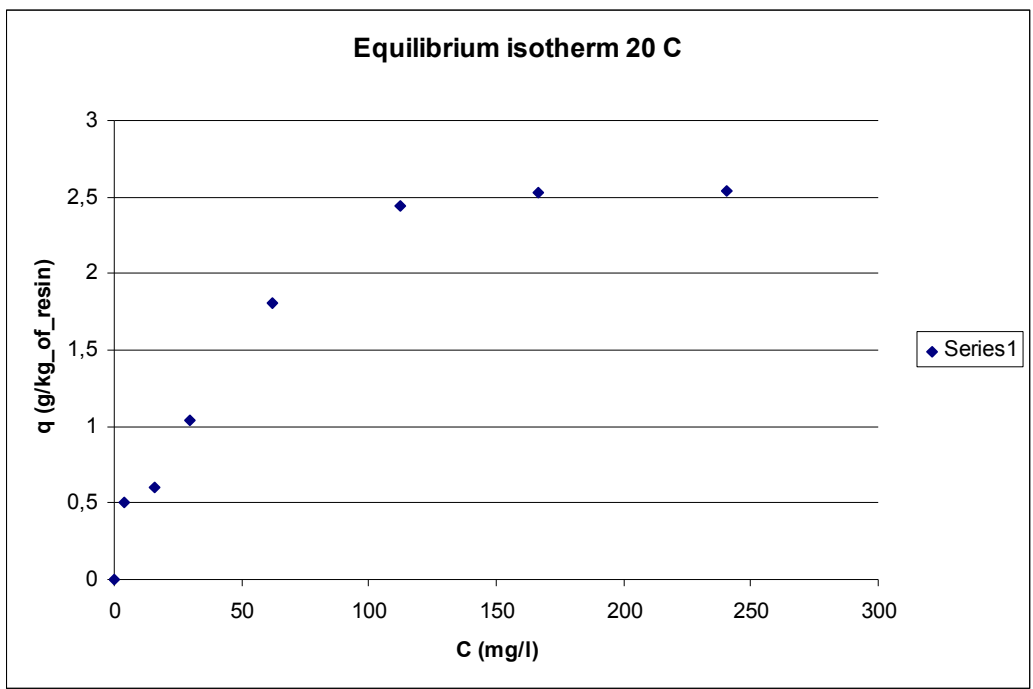

Figure 3: $\quad$ Sorption isotherm for $\mathrm{SH}($ grain size $<100 \mu \mathrm{m})$ at $19 \pm 1{ }^{\circ} \mathrm{C}$.

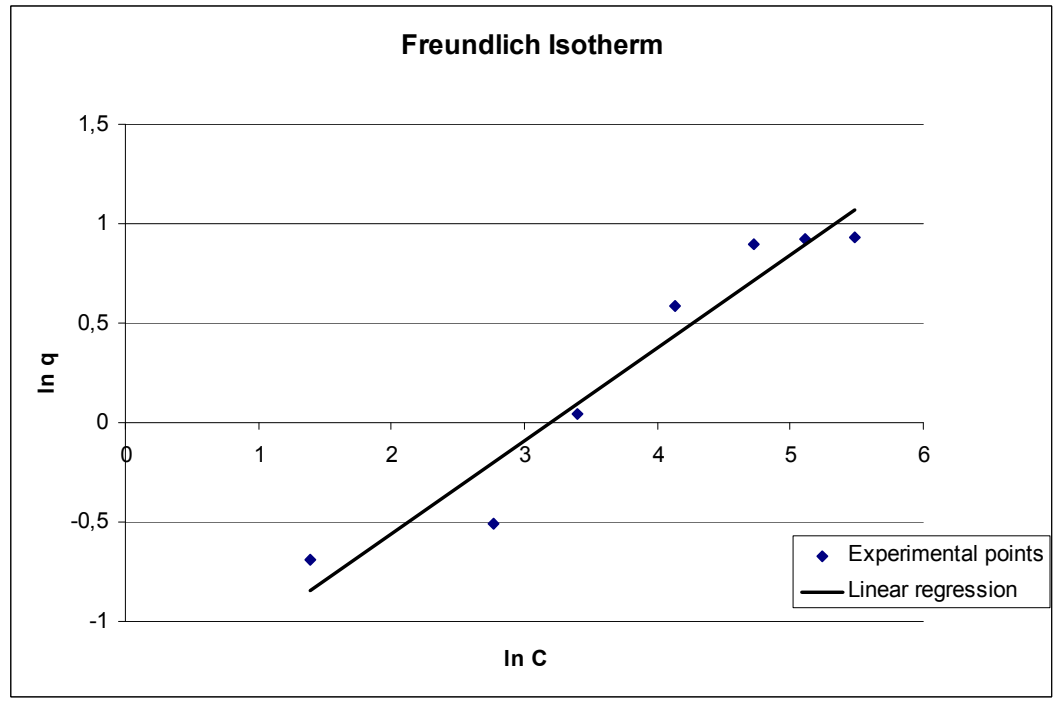

Figure 4: Linear correlation between sorbent loading and solution concentration when expressed with their natural logarithm (R2=0.93).

The Freundlich isotherm has already been mentioned in eqn. (4), and it was introduced to describe sorption equilibrium. Parameters $K$ and $n$ are Freundlich constant and Freundlich exponent, respectively, and the two together incorporate all factors affecting the sorption process such as sorption capacity and intensity 
(Rengaraj et al. [22]). The linear plot of $\log \left(q_{e}\right)$ vs $\log \left(C_{e}\right)$ shows that the adsorption follows Freundlich model, and $n(0.47)$ and $K\left(0.23(\mathrm{~g} / \mathrm{kg}) \cdot\left(\mathrm{m}^{3} / \mathrm{g}\right)^{\mathrm{n}}\right)$ could be calculated from the intercept and slope of the plot (Figure 4).

\subsection{Prediction of breakthrough}

In Figure 5 the results of the batch experiment pursued for kinetics determination are given. The drawn line shows the predicted value accordingly to the LDF model, with the mass transfer coefficient $k$ as the only fitting parameter.

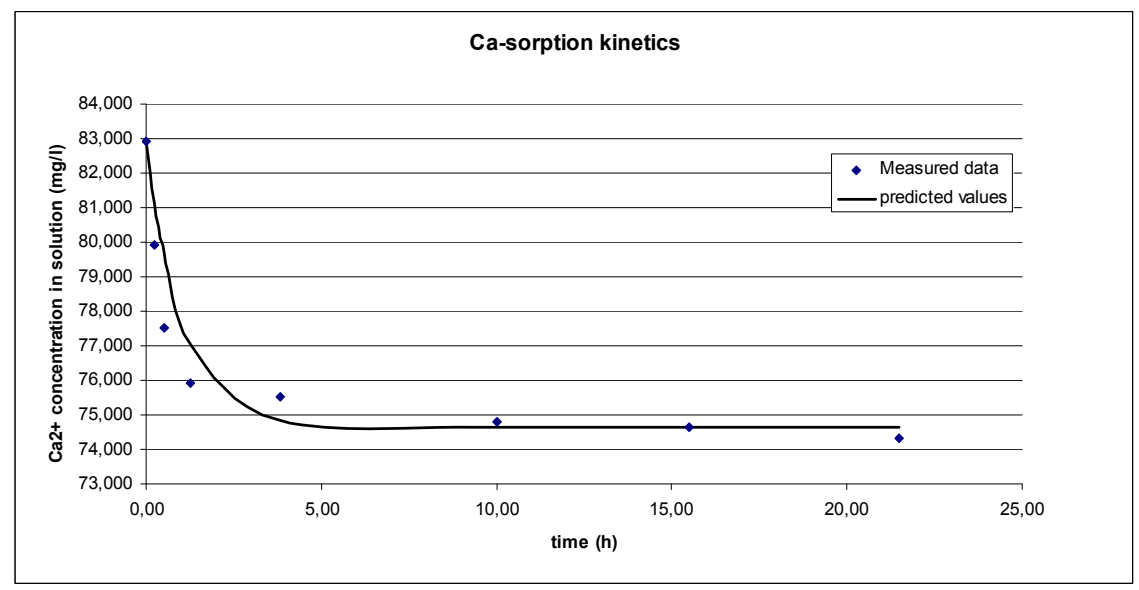

Figure 5: Decrease in concentration during the softening process with $\mathrm{SH}$ (grain size $250-500 \mu \mathrm{m}$ ) at $19 \pm 1^{\circ} \mathrm{C}$.

From the previously discussed batch experiments, conducted with full-size grains and concentrations similar to the one used in the column experiments, a precise set of parameters could be obtained in order to predict the expected breakthrough.

The model was developed in Matlab based on the one introduced by Heijman et al. [25], which develops from the mass balance of the target species in the column as shown in eqn. (7)

$$
\frac{\partial C}{\partial t}=D_{L} \frac{\partial^{2} C}{\partial z^{2}}-v \frac{\partial C}{\partial z}-\left(\frac{1-\varepsilon}{\varepsilon}\right) \rho \frac{\partial q}{\partial t}
$$

where $v$ is the superficial velocity of the water phase in the packed bed, $D_{L}$ the dispersion, $\varepsilon$ the void fraction between the adsorbent and $\rho$ the density of the adsorbent. The accumulation of the species on the hydrogel $(\partial q / \partial t)$ was described through the LDF model, as presented earlier. The equations were discretized in space by assuming the column as a series of six completely mixed unit elements with constant size and were numerically integrated in time. Axial dispersion was not accounted in the preliminary modeling. Results are shown in Figure 6. 


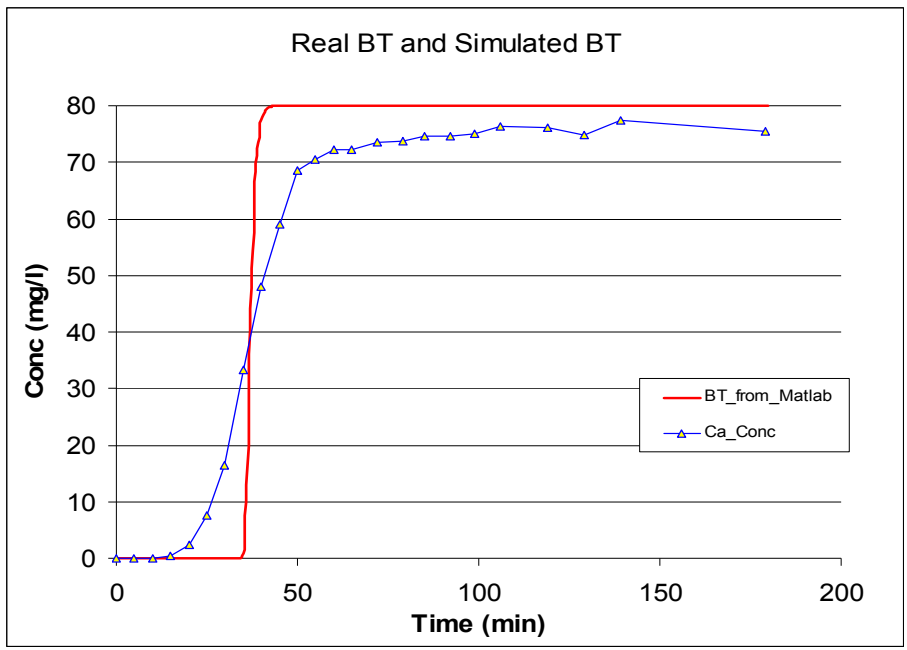

Figure 6: $\quad$ Measured and predicted breakthrough curve for a small column experiment, at $20^{\circ} \mathrm{C}$.

As observable from the presented graph, where obtained results were plotted together with the observed breakthrough of a small column, the model built was effective in predicting the breakthrough time, defined as the time when the ratio $\mathrm{C}_{\text {effluent }} / \mathrm{C}_{0}$ equals 0.5 (van Halem et al. [27]).

\section{Conclusions and further research}

At a first glance, the parameters obtained by the isotherm construction, especially the $K$, have quite a low value, compared to the ones normally found in water treatment. A maximum capacity of $0.15 \mathrm{meq} \mathrm{Ca}^{2+} / \mathrm{g}$ of dry gel is reached. According to the producer (Afira BV, The Netherlands) $0.794 \mathrm{mmol}$ of single charged anionic sites per gram of resin should be present. In this scenario, the theoretical capacity of the resin is more than 5 times the one achieved during the experiments, so less than $20 \%$ of built-in capacity is effectively used. Working on the polymer synthesis in order to obtain a better positioning of the charged groups within the resin matrix can still bring a substantial improvement. It is by the way important to take into account that the process object of the present investigation has no relation with traditional treatments, and lower $K$ should be expected. It will be important to have some more information about the necessary $K$ value (strictly linked with the overall capacity of the material) for an economically feasible process.

\section{References}

[1] C. Gabrielli, G. Maurin, H. Francy-Chausson, P. Thery, T.T.M. Tran, M. Tlili, Electrochemical water softening: principle and application, Desalination, 201 (2006) 150-163. 
[2] J.S. Park, J.H. Song, K.H. Yeon, S.H. Moon, Removal of hardness ions from tap water using electromembrane processes, Desalination, 202 (2007) 1-8.

[3] S.-J. Seo, H. Jeon, J.K. Lee, G.-Y. Kim, D. Park, H. Nojima, J. Lee, S.-H. Moon, Investigation on removal of hardness ions by capacitive deionization (CDI) for water softening applications, Water Research, 44 (2010) 22672275.

[4] S. Ghizellaoui, A. Chibani, S. Ghizellaoui, Use of nanofiltration for partial softening of very hard water, Desalination, 179 (2005) 315-322.

[5] P. Čuda, P. Pospíšil, J. Tenglerová, Reverse osmosis in water treatment for boilers, Desalination, 198 (2006) 41-46.

[6] A. Hatefi, B. Amsden, Biodegradable injectable in situ forming drug delivery systems, Journal of Controlled Release, 80 (2002) 9-28.

[7] I. Mikšík, P. Sedláková, K. Mikulíková, A. Eckhardt, T. Cserhati, T. Horváth, Matrices for capillary gel electrophoresis - a brief overview of uncommon gels, Biomedical Chromatography, 20 (2006) 458-465.

[8] F.G. Helfferich, Ion Exchange, McGraw-Hill, New York, 1962.

[9] D.J. Beebe, J.S. Moore, J.M. Bauer, Q. Yu, R.H. Liu, C. Devadoss, B.-H. Jo, Functional hydrogel structures for autonomous flow control inside microfluidic channels, Nature, 404 (2000) 588-590.

[10] S.K. De, N.R. Aluru, B. Johnson, W.C. Crone, D.J. Beebe, J. Moore, Equilibrium swelling and kinetics of pH-responsive hydrogels: models, experiments, and simulations, Microelectromechanical Systems, Journal of, 11 (2002) 544-555.

[11] M.V. Deshmukh, A.A. Vaidya, M.G. Kulkarni, P.R. Rajamohanan, S. Ganapathy, LCST in poly(N-isopropylacrylamide) copolymers: high resolution proton NMR investigations, Polymer, 41 (2000) 7951-7960.

[12] L.D. Taylor, L.D. Cerankowski, Preparation of Films Exhibiting a Balanced Temperature Dependence to Permeation by Aqueous Solutions - a Study of Lower Consolute Behavior, J Polym Sci Polym Chem Ed, 13 (1975) 25512570 .

[13] M. Heskins, J.E. Guillet, Solution Properties of Poly(Nisopropylacrylamide), J. Macromol. Sc., Chem, 2 (1968) 1441 - 1455.

[14] L.M. Geever, D.M. Devine, M.J.D. Nugent, J.E. Kennedy, J.G. Lyons, A. Hanley, C.L. Higginbotham, Lower critical solution temperature control and swelling behaviour of physically crosslinked thermosensitive copolymers based on N-isopropylacrylamide, European Polymer Journal, 42 (2006) 2540-2548.

[15] H. Kanazawa, K. Yamamoto, Y. Matsushima, N. Takai, A. Kikuchi, Y. Sakurai, T. Okano, Temperature-Responsive Chromatography Using Poly(N-isopropylacrylamide)-Modified Silica, Analytical Chemistry, 68 (1996) 100-105.

[16] J.P.A. Custers; R. J. Sablong; F. A. M. Leermakers; J. T. F. Keurentjes; D.T.A. Van Asseldonk, Process for Separating a Charged Species from an Aqueous System, in WO 2009/028944, 2009. 
[17] P.M. Sharples, B.A. Bolto, Desalting in Australia : The development of a new process for brackish water, Desalination, 20 (1977) 391-401.

[18] W. Cai, R.B. Gupta, Thermosensitive and ampholytic hydrogels for salt solution, Journal of Applied Polymer Science, 88 (2003) 2032-2037.

[19] W.F. Lee, R.J. Chiu, Thermoreversible Hydrogels. XVIII. Synthesis, Swelling Characteristics, and Diffusion Behaviors of Porous, Ionic, Thermosensitive Hydrogels, Journal of Applied Polymer Science, 90 (2003) 2214-2223.

[20] R.O. Dunn Jr, J.F. Scamehorn, S.D. Christian, Simultaneous removal of dissolved organics and divalent metal cations from water using micellarenhanced ultrafiltration, Colloids and Surfaces, 35 (1989) 49-56.

[21] G. McKay, Adsorption of dyestuffs from aqueous solutions with activated carbon I: equilibrium and batch contact-time studies, Journal of chemical technology and biotechnology, 32 (1982) 759-772.

[22] S. Rengaraj, J.W. Yeon, Y. Kim, Y. Jung, Y.K. Ha, W.H. Kim, Adsorption characteristics of $\mathrm{Cu}(\mathrm{II})$ onto ion exchange resins $252 \mathrm{H}$ and $1500 \mathrm{H}$ : Kinetics, isotherms and error analysis, Journal of Hazardous Materials, 143 (2007) 469-477.

[23] E. Glueckauf, J.I. Coates, Theory of chromatography. Part IV. The influence of incomplete equilibrium on the front boundary of chromatograms and on the effectiveness of separation, Journal of the Chemical Society (Resumed), (1947) 1315-1321.

[24] R.G. Peel, A. Benedek, A simplified driving force model for activated carbon adsorption, The Canadian Journal of Chemical Engineering, 59 (1981) 688-692.

[25] S.G.J. Heijman, W. Siegers, R. Sterk, R. Hopman, Prediction of breakthrough of pesticides in GAC-filters and breakthrough of colour in ion-exchange-filters, in: Water Science and Technology: Water Supply, 2002, pp. 103-108.

[26] A.M. Wachinski, Ion Exchange Treatment for Water, American Water Works Association, 2006.

[27] D. van Halem, S. Olivero, W.W.J.M. de Vet, J.Q.J.C. Verberk, G.L. Amy, J.C. van Dijk, Subsurface iron and arsenic removal for shallow tube well drinking water supply in rural Bangladesh, Water Research. 